Archives de sciences sociales des religions

148 | octobre-décembre 2009

Bulletin Bibliographique

\title{
La thèse de Tarot-Tesnière
}

Jean-Claude Dumoncel

\section{Q OpenEdition \\ Journals}

Édition électronique

URL : http://journals.openedition.org/assr/21478

DOI : $10.4000 /$ assr. 21478

ISSN : $1777-5825$

Éditeur

Éditions de l'EHESS

Édition imprimée

Date de publication : 31 décembre 2009

Pagination : 65-71

ISBN : 978-2-7132-2218-4

ISSN : 0335-5985

Référence électronique

Jean-Claude Dumoncel, «La thèse de Tarot-Tesnière », Archives de sciences sociales des religions [En ligne], 148 | octobre-décembre 2009, mis en ligne le 01 janvier 2013, consulté le 19 avril 2019. URL : http://journals.openedition.org/assr/21478; DOI : 10.4000/assr.21478 


\section{Jean-Claude Dumoncel}

\section{La thèse de Tarot-Tesnière}

Le livre de Camille Tarot sur les théories de la religion, par l'ampleur du débat qu'il ouvre avec la plupart des plus grands auteurs sur le sujet, par la maîtrise avec laquelle il permet à ce débat de se déployer dans tout son développement, et, surtout, par la nouvelle conception vers laquelle il conduit, une fois le débat parvenu à son terme, est certainement une nouvelle donne dans sa discipline ${ }^{1}$. Faire émerger l'économie conceptuelle sous-jacente qui dévoile dans ce travail ses plus riches perspectives heuristiques est pour le philosophe une entreprise d'un intérêt inépuisable, qui sera seulement esquissée ici.

Le point de départ le plus suggestif nous est donné par la convergence de deux propositions. L'une est de Tarot lecteur de Mauss. Exposant la théorie du sacrifice de Hubert et Mauss, il écrit (2003 : 42) : "Le sacrifice apparaît comme une sorte de dramaturgie. » Ce faisant, Tarot donne son aval à Hubert et Mauss eux-mêmes, qui ont décrit le sacrifice comme un "drame " $(1899$ : 212). Il s'agit donc, d'abord, d'une thèse d'Hubert et Mauss, que j'appellerai aussi thèse de Tarot en tant qu'elle est intégrée à sa description systématique de la religion (2008b). L'autre proposition est celle où Lucien Tesnière (1959:102) a condensé l'idée-clef de sa Syntaxe structurale. Selon lui, "le nœud verbal » qui contient d'après sa théorie la «structure de la phrase simple » exprime tout un petit drame.

La convergence que nous évoquons est renforcée quand on entre dans le détail. Le drame du sacrifice a, selon Tarot, "des acteurs " (2003: 42) dont Hubert et Mauss proposent une liste en distinguant Sacrifiant, Sacrificateur et Victime (1899: 212). À quoi il faut ajouter un lieu (comme le temple) et un temps (opportun) (1899: 221). De même, pour fonder sa vision du nœud verbal comme drame, Tesnière note : "Comme un drame en effet, il comporte obligatoirement un procès, et le plus souvent des acteurs et des circonstances ". Il ajoute :

1. Je dois remercier Henri Droguet qui m'a depuis longtemps signalé l'importance des travaux de Camille Tarot et qui m'a permis de le rencontrer.

ARCHIVES DE SCIENCES SOCIALES DES RELIGIONS 148 (octobre-décembre 2009), pp. 65-71 
«Transposés du plan de la réalité dramatique sur celui de la syntaxe structurale, le procès, les acteurs et les circonstances deviennent respectivement le verbe, les actants et les circonstants. »

Relativement à la description du sacrifice par Hubert et Mauss, qui met déjà en scène un sacrifiant, une victime et un sacrificateur, le drame de Tesnière demeure dans l'abstrait. Si nous voulons maintenir notre parallèle, nous devons remédier dès que possible à ce déséquilibre. À cette fin, nous allons pénétrer dans un nouveau parallèle, dû à Vincent Descombes dans ses Essais sur le Don (1996, chap. 18). Conduit à s'intéresser aux relations triadiques, Descombes a découvert que celles-ci demandaient une double attaque, logique et linguistique, dont se sont chargés respectivement Peirce et Tesnière. Le philosophe américain C. S. Peirce a été l'un des principaux fondateurs de la logique des relations, ce qui l'a conduit, entre autres, à démontrer que les relations à trois termes (comme "A sacrifie un $\mathrm{B}$ à $\mathrm{C}$ ») sont irréductibles à des combinaisons de relations binaires. Et pour Peirce, le paradigme de la relation triadique n'est autre que le Don (1904 : 28). Sa forme logique se révèle dans la fonction propositionnnelle : A donne B à C.

Mais les termes A, B et C n'y sont pas des variables interchangeables. Chacun représente un rôle différent correspondant à sa place dans la relation. Dans le Don analysé selon le vocabulaire de Descombes, A est le Donateur, B le Don (nous dirons aussi le présent) et C le Donataire. Dans le drame du Don, les actants sont maintenant distingués au même étiage que dans le drame du sacrifice. Ce qui permet en retour de déceler un oubli de Mauss dans son inventaire des rôles sacrificiels : à savoir le dédicataire. Socrate devait un coq à Esculape. Mais ce que Peirce vient de traiter en compréhension, Tesnière (1959: 255) va l'aborder en extension dans une observation quelque peu énigmatique relevée aussi par Descombes : "Les verbes à trois actants sont en principe des verbes de dire et de don ». Tesnière ajoute une liste des principaux, où nous relèverons seulement raconter, jurer et confesser pour le dire, destiner, promettre, payer, rendre, et aussi sacrifier pour le don.

Le Symbole et le Sacré, c'est aussi une explication avec le structuralisme. Et sur le structuralisme, deux surprises nous attendent.

1- La typologie des relations de Saussure (1916, $2^{\mathrm{e}}$ partie, chap. V) est $\grave{a}$ l'étiage ontologique de Peirce: les relations paradigmatiques et syntagmatiques ne sont pas illustrées seulement par les langues ni même les colonnes grecques. La table de Mendeleïev est un paradigme, une molécule un syntagme.

2- Frazer est le fondateur du structuralisme. Comme Tarot l'a vu (2003: 44) le distinguo de Frazer entre magie homéopathique et contagieuse illustre la pertinence de l'opposition Métaphore/Métonymie à l'échelle de la culture entière, et donc au premier chef sur la religion.

Chacune des deux propositions ainsi rapprochées demande avant tout à être généralisée comme le distinguo paradigme/syntagme. Pour la thèse de Tarot, la 
généralisation va de soi. Si le sacrifice est une dramaturgie, alors il en va de même de toute cérémonie religieuse et donc dans une certaine mesure de tout rite. Comme le dira Mauss lui-même (1947 : 167) : tout rituel est essentiellement dramatique.

Si on hésite à parler de dramaturgie dans le cas d'une simple bénédiction, par exemple, on pourra s'inspirer ici de Tesnière pour parler de "petite dramaturgie ». Le point principal est que le caractère dramaturgique n'est pas confiné au sacrificiel mais porte sur le culte en totalité. Pour la thèse de Tesnière, la généralisation demande une transposition théorique dont le principe a été posé par Jean Petitot (Petitot-Cocorda, 1985). On sait que depuis Lévi-Strauss (1955), l'analyse structurale des mythes se réclame d'une inspiration linguistique. Cette analyse structurale des mythes se situe par ailleurs dans une entreprise plus vaste et plus ancienne qui est l'analyse structurale du récit. Petitot (ibid.) remarque :

"Lorsque l'on considère les corpus constitués, par exemple, de mythes, de contes merveilleux, de tragédies, de romans, etc., l'on constate aussitôt qu'au niveau superficiel (apparent), ces récits racontent des intrigues entre des personnages (des acteurs) localisés et déplacés spatio-temporellement, définis par des rôle thématiques, liés par des rapports d'état civil, ou contractuels, ou passionnels et interagissant entre eux à travers des conflits, des dons, des contrats, des séparations, des retrouvaille, etc. Audelà de la diversité foisonnante des structures discursives superficielles, on peut faire apparaitre des structures profondes, appelées par A. J. Greimas structures sémionarratives. »

Plus précisément :

«La reconnaissance d'une composante syntaxique de la grammaire sémio-narrative profonde remonte aux travaux de Propp sur le conte russe, exposés dans son ouvrage célèbre La morphologie du conte. Sous-jacentes aux diverses actions des personnages du récit, Propp a dégagé des fonctions (c'est-à-dire des relations actantielles typiques) enchaînées canoniquement, se développant de façon réglée comme un processus de morphogenèse, et s'exprimant dans des séquences du type : établissement d'un manque initial (par exemple transgression des règles sociales, trahison, etc.), contrat entre un Destinateur (par exemple roi, groupe social, etc.) et un héros, succession d'épreuves, d'abord épreuves qualifiantes au cours desquelles le héros acquiert des modalités de vouloir, de savoir et/ou de pouvoir (par exemple obtention d'une arme magique par l'intermédiaire d'un adjuvant), ensuite épreuve décisive où le héros accomplit une performance liquidant le manque initial (par exemple en tuant un dragon), enfin épreuve glorifiante où la performance du héros se trouve sanctionnée par le Destinateur. Autrement dit, Propp a repéré et formulé des structures actantielles invariantes, stables et universelles, régies par une syntaxe actantielle qui syntagmatise... un paradigme actantiel constitué des relations Destinateur/Destinataire, Sujet/Objet de valeur, Sujet/Antisujet, Adjuvant/Opposant. »

La fonction, donnée dans cette perspective à la notion d'actant et relevée par Tarot (2008a: 493) ${ }^{2}$, désigne l'articulation conceptuelle de la syntaxe structurale due à Tesnière et de la morphologie du récit due à Propp. Se définit à partir

2. Tarot évoque là les " actants de Greimas ". 
de là un Morphologie du Mythe où l'axiome-clé sera: le mythe est un drame de Tesnière.

Aussitôt cette généralisation acquise, elle appelle équilibrage en sens inverse de celui qui portait sur la proposition de Tesnière. Un des points forts du modèle de Tarot est la place qu'il a su donner sur la religion à la découverte de la dimension pragmatique (2008a : 86-88) du langage par la philosophie analytique, sous l'impulsion principale de J. L. Austin dans Quand dire, c'est faire (1962). Or cette découverte est, depuis la percée d'Oswald Ducrot, en 1972, dans un rapport de symbiose exemplaire avec la sociologie de Mauss. Dans son Essai sur le Don de 1924 (1950 : 166-167), Mauss nous offre un paradigme du rite :

«Ce n'est pas seulement pour manifester puissance et richesse et désintéressement qu'on met à mort des esclaves, qu'on brûle des huiles précieuses, qu'on jette des cuivres à la mer, qu'on met même le feu à des maisons princières. C'est aussi pour sacrifier aux esprits et aux dieux, en fait confondus avec leurs incarnations vivantes, les porteurs de leurs titres, leurs alliés initiés. »

Plus abstraitement :

«Les rapports de ces contrats et échanges entre hommes et de ces contrats et échanges entre hommes et dieux éclairent tout un côté de la théorie du Sacrifice. »

Dans la typologie des actes de langage distingués par Austin, l'essentiel est la différence entre un acte illocutoire comme marchander et un acte perlocutoire comme faire baisser le prix. Ducrot (1972 : 78-79) a découvert que ce qui fait la différence entre ces actes d'Austin est dit par Mauss dans son analyse du potlatch. Le don change la relation juridique du donateur au donataire en créant pour le donataire une obligation en retour. De même, alors que l'acte perlocutoire n'est que l'effet (plus ou moins mécanique) d'une cause, l'acte illocutoire modifie le rapport juridique des interlocuteurs. "Auriez-vous l'heure ? crée une obligation de réponse. Celui à qui on marchande ne peut plus que jouer le jeu du marché. Etc.

En éclaircissant le concept d'illocutoire, Ducrot prévient du même coup le contresens le plus facile à faire sur la théorie d'Austin, qui la réduit à un distinguo entre langage "constatif» (du type «Il neige ») et langage "performatif» (du type "Je te baptise»). En réalité toute parole inclut un acte illocutoire (par exemple l'assertion pour "Il neige »). Mais on aura remarqué que les exemplestypes de performatif les plus parlants ("Je te baptise ", "Je vous déclare mariés») sont autant d'exemples de rites. Or, si on admet avec Mauss (19021903 : 53) que «tout rite est une espèce de langage ", il s'ensuit que tout rite est du langage performatif. L'équilibrage à obtenir est obtenu. La prise en considération de la pragmatique par Tarot assure à sa théorie l'entrée la plus appropriée sur le rite.

Nous appellerons thèse de Tarot-Tesnière la thèse affirmant que les mythes sont des drames de Tesnière à décrire dans une Morphologie du Mythe de même que les rites sont des drames de Mauss à penser comme "performances » d'Austin. 
La théorie de la religion dont cette thèse est le pivot est donc une description $d u o$ dramatique de la religion. Tarot (2008a : 691) définit la religion comme le Système Symbolique du Sacré. Cette définition condense deux thèses capitales de Durkheim (1912 : 49-51) au moyen d'un concept provenant aussi de Durkheim. Selon les deux thèses: 1- La religion se définit par l'opposition du Sacré au profane ; 2- Le fait religieux se diffracte d'emblée « en deux catégories fondamentales " : les rites et les mythes (que Durkheim conçoit très clairement comme cas particuliers de la différence entre action et représentation). Le concept condensateur (Tarot, 2008a : 285) est celui de symbole illustré par un paradigme de Durkheim (1912 : 314-315) : le totem est le drapeau du clan. Car l'animal totem figure à la fois dans le mythe et dans le rite.

Ainsi, dans la perspective ouverte par Tarot, mythe et rite sont subsumés deux fois. La première fois par le Symbolique, dans la définition que nous venons de développer. C'est l'approche "aristotélicienne ", où une science vise avant tout une définition de son objet. Mais le problème est, à partir de là, de déterminer comment cette définition s'articulera sur une véritable théorie, capable d'explorer le territoire ainsi défini ; c'est l'approche "poppérienne ». Là, mythe et rite sont subsumés une seconde fois par la conception duo-dramatique. L'idée de "drame » s'y dédouble pour épouser au plus près la diffraction du symbole religieux en drame narratif et drame cérémoniel. L'inclusion des conquêtes spéculatives de la pragmatique par Tarot s'y prolonge naturellement par l'inclusion des conquêtes narratologiques du même étiage théorique.

Du côté rite, la convergence conceptuelle dégagée par Ducrot entre Austin et Mauss est exemplaire. Cependant, il arrive que le développement technique demande à être dominé par une vision moins détaillée mais plus vaste. C'est ce qui fait l'intérêt de l'identité posée par Ducrot (1972: 99) entre ce que Mauss appelle "échange ", et Wittgenstein, " jeu ». Une telle équation ne doit pas être prise au pied de la lettre. Mais elle éclaire la totalité de notre sujet. On sait que chez le second, l'investigation philosophique se développe à partir d'un couple conceptuel où les deux termes sont des jeux de langage et les formes de vie dont ils font partie. Dans la liste de jeux de langage que Wittgenstein donne comme exemples (1953: $\$ 23)$, le dernier venu est prier (beten), dont la forme de vie est évidemment la religion. Il est précédé par "inventer une histoire ». Ni la notion de « jeu " ni la «vie " de la «forme de vie » ne doivent nous illusionner sur ce que nous allons trouver là. Parmi les exemples que Mauss donne de dépenses somptuaires dans les échanges sacrificiels, il y a celles où l'on met à mort des esclaves. Et quand il s'agit du sacrifice où une Iphigénie peut être sacrifiée, il va de soi qu'il n'y a pas à fournir d'arguments pour le qualifier de drame. Ce qui nous conduit à « la catégorie définie par Frazer » selon René Girard (1978:156) et dont Tarot a vu le caractère critériologique (2008a : 338 et passim).

Dans le Lévitique (XVI), Yahvé prescrit qu'Aaron reçoive de la communauté deux boucs dont un tirage au sort choisira l'un pour Yahvé, l'autre pour le démon Azazel. Le bouc voué à Yahvé sera tué en expiation "pour le péché ", 
l'autre sera chargé de tous les péchés du peuple et lancé dans le désert. L'un est dit bouc "expiatoire ", l'autre bouc émissaire. L'un est sacrifié, l'autre exilé. Cuvillier (1962 : $\$ 169)$ décrit le rite du bouc émissaire comme l' " adoucissement probable d'une coutume plus ancienne où un individu était choisi comme victime expiatoire et chargé de tous les péchés du groupe ». La double prescription contient déjà une double substitution symbolique. D'une part, la mise à mort est commuée en exil. D'autre part, le paiement de la faute est remplacé par son déplacement. L'intérêt de l'interprétation notée par Cuvillier tient à ce qu'elle inscrit cette double substitution littérale dans une substitution latente où le bouc a été substitué à une victime humaine telle que les esclaves dont parlait Mauss. Mais à travers cet emboîtement de substitutions, une constante se maintient, c'est que la prétendue "expiation » consiste à faire expier le péché des uns par un autre qui n'y est pour rien, esclave ou bouc, tué ou exilé.

Discutant Frazer, Hubert et Mauss présupposent pour définir « le rôle » du " bouc émissaire » la capacité d' " emporter avec lui la maladie, la mort, le péché » (1899 : 196). Or il y a un abîme entre se charger du fardeau moral (moral burden) de la faute et emporter au diable la maladie et la mort. Dans le premier cas, le Bouc biblique suffit. Dans le second, il faut au minimum avoir un souffre-douleur. Du point de vue théorique, ou bien nous territorialisons sur le Lévitique, ou bien nous en faisons comme Girard un morceau de mandala, sinon le Mandala des Mandalas.

Chez Deleuze et Guattari (1980 : 169, 146-147) le bouc expiatoire et le bouc émissaire sont respectivement les termes 5 et 6 d'un Schéma de la Sémiotique Signifiante où le terme 4 est "le développement interprétatif du signifiant en signifié ». Mais quel est le signifiant dont cette interprétation cherchera le signifié ? Dans le rite du Bouc émissaire interviennent en amont deux acteurs qui ne sont autres que Yahvé et Azazel. Or, ces deux acteurs incarnent deux actants qui sont ici le dieu et le démon. Ce ne sont pas comme chez Pirandello des "personnages en quête d'auteur " mais des acteurs de Tarot en quête de leur drame de Tesnière. Autrement dit, ce sont des acteurs de rite en quête du mythe dont ils seraient les actants. Comme "Azazel " est ici un "signifiant flottant» au sens de Lévi-Strauss (1950 : XLIX) nul doute que les mythes ne manqueront pas à l'appel. Il est notoire que le premier Henoch et l'Apocalypse d'Abraham s'offrent ici à remplir la place du mythe manquant dans le canon biblique pour son rite avéré.

Il s'agissait en somme de découvrir que la description due à Tarot a les atomes crochus les mieux placés pour assurer son articulation avec les autres développements les plus solides sur son sujet. Nous espérons avoir esquissé les lignes de force principales de cet investissement heuristique.

Jean-Claude DUMONCEL

Université de Caen - Dépt de Philosophie

Jcl.dumoncel@club-internet.fr 


\section{Bibliographie}

Austin John Langshaw, 1962, How to Do Things with Words, « William James Lectures de 1955 ", Oxford, Oxford University Press.

-, 1962, Quand dire c'est faire, Paris, Seuil.

Cuvillier Armand, 1962, Manuel de sociologie, Paris, Presses Universitaires de France.

Deleuze Gilles, Guattari Félix, 1980, Mille plateaux, Paris, Éditions de Minuit.

Descombes Vincent, 1996, Les institutions du sens, Paris, Éditions de Minuit.

Ducrot Oswald, 1972, Dire et ne pas dire, Paris, Hermann.

Durkheim Émile, [1912] 1960, Les formes élémentaires de la vie religieuse, Paris, Presses Universitaires de France.

GIRARD René, 1978, [c. J.-M. Oughourlian, G. Lefort], Des choses cachées depuis la fondation du monde, Paris, Grasset.

Hubert Henri, Mauss Marcel, 1899 (cité dans Mauss, 1968), « Essai sur la nature et la fonction du sacrifice ".

LÉvi-STRAuss Claude, 1950, "Introduction à l'œuvre de Marcel Mauss », in Mauss M., Sociologie et Anthropologie, Paris, Presses Universitaires de France.

-, 1955, "La structure des mythes ", in Lévi-Strauss C., Anthropologie structurale, Paris, Plon, 1958.

Mauss Marcel, 1902-1903, "Théorie générale de la magie ", in Mauss M., Sociologie et Anthropologie, Paris, Presses Universitaires de France, 1950.

-, 1947, Manuel d'ethnographie, Paris, Payot.

-, 1950, Sociologie et anthropologie, Paris, Presses Universitaires de France.

-, 1968, Euvres 1, Paris, Minuit.

Petitot-Cocorda Jean, 1985, Morphogenèse du sens I, Paris, Presses Universitaires de France.

PeIrce Charles S., 1904, (cité dans Peirce, 1978], "Lettre à Lady Welby », in Peirce C. S., Écrits sur le signe, Paris, Seuil, 1978.

-, 1978, Écrits sur le signe, Paris, Seuil.

Saussure Ferdinand de, 1916, Cours de linguistique générale, Paris, Payot.

TArot Camille, 2003, Sociologie et anthropologie de Marcel Mauss, Paris, La Découverte. -, 2008a, Le symbolique et le sacré. Théories de la religion, Paris, La Découverte.

-, 2008b, "Modèle et fonctions de la religion ", Conférence au Centre d'études théologiques de Caen, 26 novembre.

TESNIÈRE Lucien, 1959, Éléments de syntaxe structurale, Paris, Klincksieck.

WitTGENSTEIN Ludwig, [1953] 1961, Recherches philosophiques, Paris, NRF. 
